\title{
Simulation of AntiMatter-Matter Interactions in Geant4
}

\author{
Aida Galoyan ${ }^{1, \star}$, Vladimir Uzhinsky ${ }^{2}$, and Alberto Ribon ${ }^{3}$ \\ ${ }^{1}$ VBLHEP, JINR, Dubna, Russia \\ ${ }^{2}$ LIT, JINR, Dubna, Russia \\ ${ }^{3}$ CERN, Geneva, Switzerland
}

\begin{abstract}
Cross sections of antiproton and antinucleus interactions with nuclei are calculated using stochastic averaging method. A new implementation of the QuarkGluon-String Model (QGSM) is proposed for simulation of multi-particle production in antinucleus-nucleus collisions. A combination of the cross sections and the new implementation of QGSM allows experimental data on antiproton and antinucleus interactions with nuclei to be described. The combination is included in the well-known Geant 4 simulation toolkit.
\end{abstract}

\section{Introduction}

One of the most exciting puzzles in cosmology is connected with the question of the existence of antimatter in the Universe. Some cosmic ray experiments (PAMELA, BESS, AMS, CAPRISE etc.) aim to search for antinuclei. Antinuclei have also been observed in nucleus-nucleus and protonproton collisions at accelerators by the RHIC and LHC Collaborations. An experimental study of the antinucleus production and reactions requires the knowledge of antinucleus interaction cross sections with the matter. The cross sections are needed to estimate various experimental corrections, especially those due to particle losses which reduce the detected rate. We calculate the cross sections using the Glauber approach [1-3].

Many particles (pions, K-mesons, baryons and so on) are produced in the antiproton-proton and antinucleus-nucleus interactions. To simulate their production, we use the main ideas of the QuarkGluon-String model $[4,5]$. The main ingredient of the model is the estimation of various process cross sections of the quark-gluon string productions. The simulation of the string fragmentation is based on the LUND fragmentation model. All of these allow the known experimental data to be described successfully.

The combination of these models is included in the Geant 4 simulation toolkit [6]. Geant 4 is a software toolkit for the simulation of the passage of particles through matter. It is used by a large number of experiments and projects in a variety of application domains, including high energy physics, astrophysics and space science, medical physics and radiation protection.

\footnotetext{
^e-mail: galoyan@1xpub01.jinr.ru
} 


\section{Cross section of antinucleus-nucleus interactions}

In the Glauber approach, the amplitude of the elastic scattering of an antinucleus containing $B$ antibaryons on a target nucleus with the mass number $A$ is given as:

$$
\begin{gathered}
F_{B A}(\mathbf{q})=\frac{i}{2 \pi} \int d^{2} b e^{i \mathbf{q} \mathbf{b}}\left\{1-\prod_{i=1}^{B} \prod_{j=1}^{A}\left[1-\gamma\left(\mathbf{b}+\tau_{\mathbf{i}}-\mathbf{s}_{\mathbf{j}}\right)\right]\right\}\left|\Psi_{B}\right|^{2}\left|\Psi_{A}\right|^{2}\left(\prod_{i=1}^{B} d^{3} t_{i}\right)\left(\prod_{j=1}^{A} d^{3} r_{j}\right) \\
=i \int_{0}^{\infty} b P_{B A}(b) J_{0}(q b) d b
\end{gathered}
$$

where $\gamma$ is the amplitude of the elastic antinucleon-nucleon scattering in the impact parameter representation averaged over the spin and isospin degrees of freedom, $\gamma(\mathbf{b})=\frac{1}{2 \pi i} \int d^{2} q e^{i \mathbf{q b}} F_{\bar{N} N}(\mathbf{q})$. $\Psi_{A}\left(\Psi_{B}\right)$ is the wave function of the nucleus (antinucleus) in the ground state. Taking the origins of the coordinate systems at the centers of the nuclei, the nucleon coordinates $\left(\left\{\mathbf{r}_{\mathbf{A}}\right\},\left\{\mathbf{t}_{\mathbf{B}}\right\}\right)$ are decomposed into longitudinal $\left(\left\{z_{i}\right\}\right)$ and transverse $\left(\left\{\mathbf{s}_{\mathbf{j}}\right\},\left\{\tau_{\mathbf{i}}\right\}\right)$ components. The $z$-axis is directed along the projectile momentum. b is the impact parameter vector orthogonal to the momentum. $P_{B A}(b)$ is the profile function and $J_{0}$ is the Bessel function of zeroth order.

Quite often $\gamma$ is parameterized as $\gamma(\mathbf{b})=\sigma_{\bar{N} N}^{\text {tot }}(1-i \rho) /(4 \pi \beta) \cdot e^{-\mathbf{b}^{2} / 2 \beta}$, where $\sigma_{\bar{N} N}^{\text {tot }}$ is the total cross section of the antinucleon-nucleon interactions, $\rho$ is the ratio of the real to imaginary parts of the $\bar{N} N$ elastic scattering amplitude at zero momentum transfer, and $\beta$ is the slope parameter of the $\bar{N} N$ differential elastic scattering cross section.

Using these expressions, one can calculate various quantities characterizing the antinucleusnucleus interactions: total, elastic and inelastic cross sections and so on (see details in [7]). In Fig. 1, the experimental absorption cross sections of the antiproton and antideuteron interactions with various nuclei are presented and compared with our calculations. As one can see, there is a good agreement between the calculations and the experimental data.
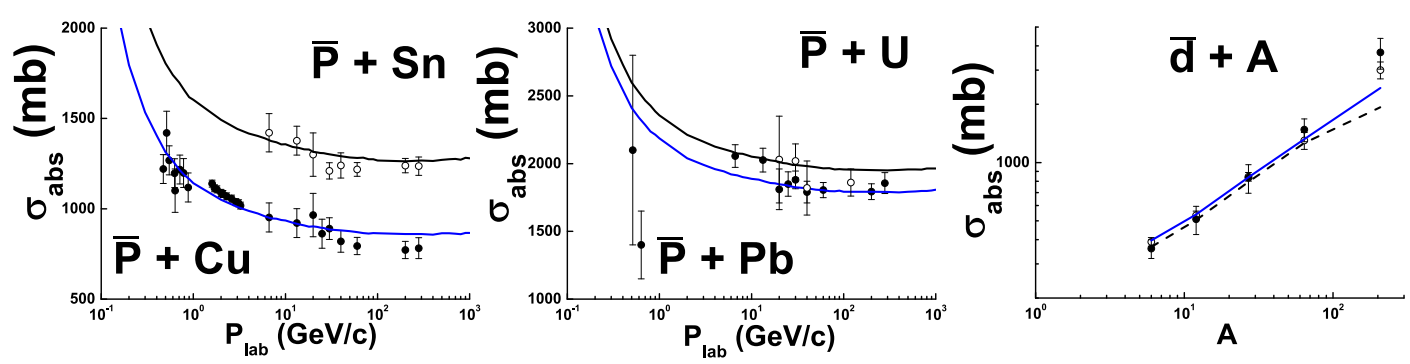

Figure 1. Absorption cross sections of the antiproton and antideuteron interactions with nuclei. Points are experimental data. The lines are our calculations. The experimental data for $\bar{d}+A$ interactions [8] are given for two momenta -13.3 and $25 \mathrm{GeV} / \mathrm{c}$ (open and close circles, correspondingly). The calculation results at the energies are presented by solid and dashed lines.

The complexity of the Glauber expressions prevents their direct calculations. A way to the solution is offered by the stochastic averaging method [9] in which a quantity is found as an average over various samples of the nucleon coordinates. The nucleon coordinates are sampled according to the measure $\left|\Psi_{A}\right|^{2}\left|\Psi_{B}\right|^{2}$. The method consists of multiple sampling of the nucleon coordinates according to the function $\left|\Psi_{A}\right|^{2}\left|\Psi_{B}\right|^{2}$, calculation of needed expression for each sampling, and an averaging of the calculation results over the samples. Results obtained in such manner were parameterized, and the parameterizations were implemented in Geant 4 . 


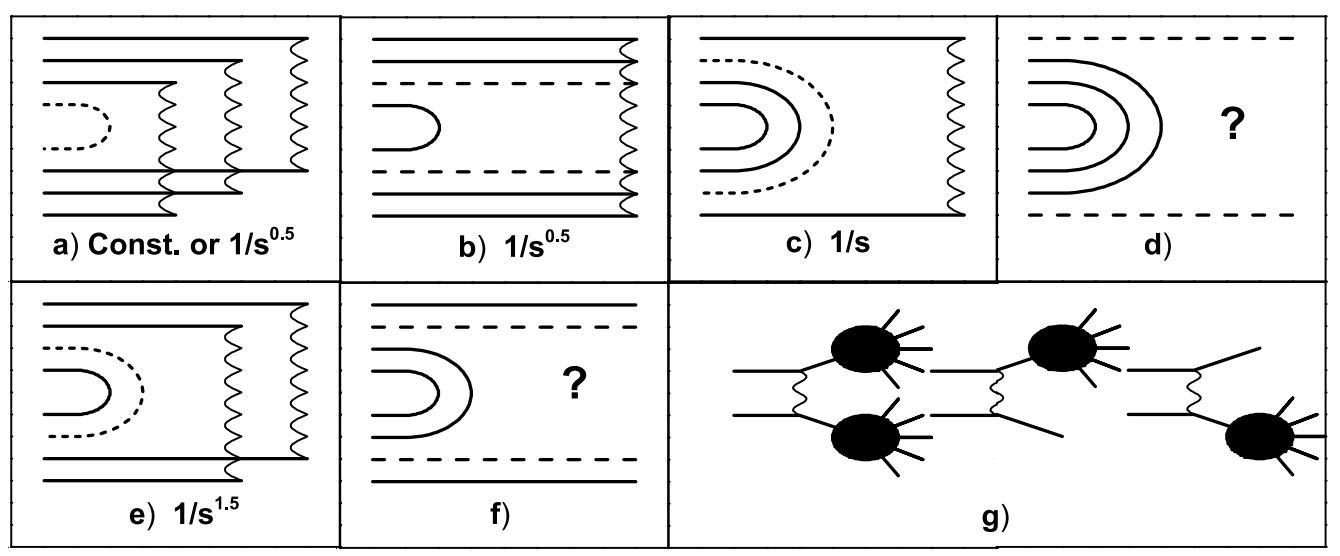

Figure 2. Quark flow diagrams of $\bar{p} p$ interactions

\section{Particle production in antinucleus-nucleus interactions}

Usually, the list of diagrams shown in Fig. 2 is considered in the high energy phenomenology for antiproton-proton interactions. The diagram $a$ corresponds to string junction annihilation with 3 quark-antiquark string creation. The string junction is a gluonic object which couples together the quarks in a baryon. The diagram $b$ represents the quark-antiquark annihilation leading to a diquarkantidiquark string creation. The diagram $c$ shows a process of diquark-antidiquark annihilation and one quark-antiquark string formation. The diagram $e$ corresponds to quark-antiquark and string junction annihilations with the creation of 2 quark-antiquark strings. The diagrams $d$ and $f$ are responsible for exotic meson production. The last diagrams at the bottom of the figure are important at high energies. They are connected with the pomeron exchange in the t-channel. The energy dependencies of the cross sections of the processes are predicted in some cases by the reggeon phenomenology. They are also shown in the figure. An elaborated scheme for the calculation of the cross sections in the reggeon phenomenology was proposed in $[10,11]$. As shown there, the approach is valid at $P_{\text {lab }}>$ $3 \mathrm{GeV} / \mathrm{c}$. Since the experimental studies require much less energies, we undertook a new attempt to estimate the cross sections. It is presented in [12].

$$
\begin{gathered}
\sigma_{a}=\frac{16}{\sqrt{s-4 m^{2}}}\left[\left(s-4 m^{2}\right)^{-0.175}+3.125 \cdot(1-1.88 / \sqrt{s})\right] \quad(\mathrm{mb}), \\
\sigma_{b}=3.13+140 \cdot\left(M_{\mathrm{th}}-\sqrt{s}\right)^{2.5} \quad(\mathrm{mb}), \quad \sqrt{s} \leq M_{\mathrm{th}}=2.172 \quad(\mathrm{GeV}), \\
\sigma_{b}=6.8 / \sqrt{s} \quad(\mathrm{mb}), \quad \sqrt{s}>M_{\mathrm{th}}=2.172 \quad(\mathrm{GeV}), \\
\sigma_{c}=23.3 / s \quad(\mathrm{mb}), \quad \sigma_{e}=\sigma_{d}=\sigma_{f}=0, \quad \sigma_{g}=35 \cdot(1 .-2.1 / \sqrt{s}) .
\end{gathered}
$$

The parameterizations (2)-(4) of the process cross sections and the LUND string fragmentation algorithm implemented in the FTF generator of Geant 4 allow one to reproduce a wide set of experimental data on the $\bar{p} p$ and $\bar{p} A$ interactions [12]. Of course, the generalization of the approach given above for the antiproton and antinucleus interactions with the nuclei is not a simple procedure. Some details of the procedure were considered in $[12,13]$. The most important issue of the moment is that we can reproduce correctly the experimental data on the antideuteron-deuteron interactions (Fig. 3).

The figure 3 plots the $\pi^{+}, \pi^{-}$and proton distributions in terms of transverse momentum squared $\left(P_{T}^{2}\right)$ respectively the rapidity $(y)$. Fig. 3 points to a very good agreement between the experimental data and our calculations. 

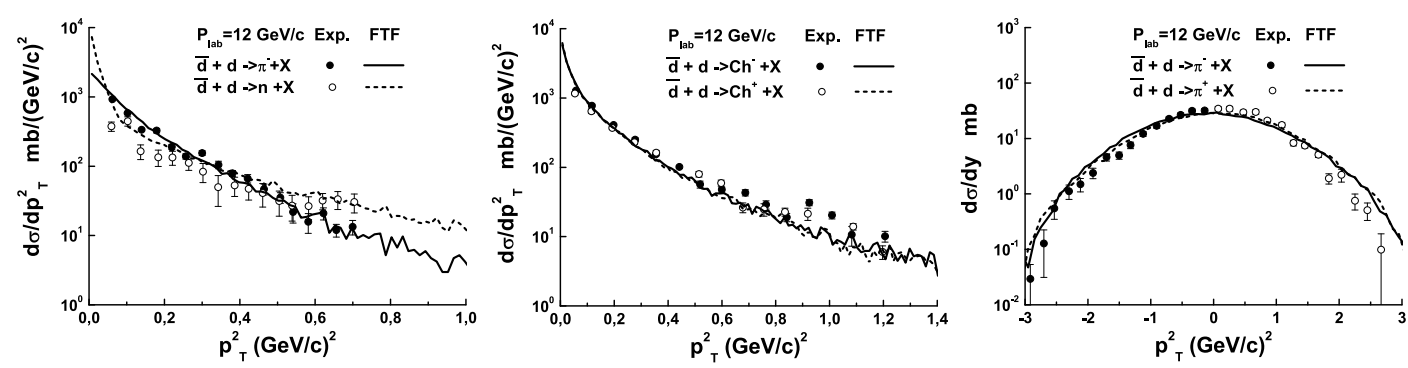

Figure 3. Distributions of particles on kinematical variables in the antideuteron-deuteron interactions at 12.2 $\mathrm{GeV} / \mathrm{c}$. Points are experimental data [14]. Lines are our calculations.

\section{Conclusion}

A new implementation of the Quark-Gluon-String model is proposed. Estimations of annihilation cross sections in the Glauber model and the LUND string fragmentation algorithm allow consistent description of a wide set of experimental data on the antiproton and antinucleus interactions with the nuclei. The proposed approach is included in the Geant 4 simulation package. It can be used in the FTF_BERT physics list of Geant4.

\section{Acknowledgement}

The authors are grateful to the heterogeneous computing team of LIT JINR (HybriLIT) for support of our calculations and to Professor Cheuk-Yin Wong for helpful discussions.

\section{References}

[1] R.J. Glauber, Lectures in Theoretical Physics (Ed. W.E.Brittin et al., v. 1, Interscience Publishers, N.Y., 1959)

[2] R.J. Glauber, Proc. of the 2nd Int. Conf. on High Energy Physics and Nuclear structure (Ed. G.A.Alexander, Rehovoth, North-Holland, Amsterdam, 1967)

[3] V. Franco, Phys. Rev. 175, 1376 (1968)

[4] A. Capella, U. Sukhatme, C-I. Tan, and J. Tran Thanh Van, Phys. Rept. 236, 225 (1994)

[5] A.B. Kaidalov and K.A. Ter-Martirosian, Phys. Lett. B117, 247 (1982)

[6] Geant4 Collaboration (J. Allison et al.), Nucl. Instrum. Meth. A835, 186 (2016)

[7] V. Uzhinsky, J. Apostolakis, and A. Galoyan et al., Phys. Lett. B705, 235 (2011)

[8] S.P. Denisov et al., Nucl. Phys. B31 253 (1971)

[9] S. Shmakov, V. Uzhinsky, and A.Zadorozhny, Comp. Phys. Comm. 54, 125 (1989)

[10] V.V. Uzhinsky and A.S. Galoyan, arXive:0212369 [hep-ph] (2012)

[11] A. Galoian and V. Uzhinsky, AIP Conf. Proc. 796, 79 (2005)

[12] A. Galoyan, A. Ribon, and V. Uzhinsky, PoS BaldinISHEPPXXII, 049 (2015)

[13] Geant4 Hadronics Working Group (A. Galoyan et al.) Hyperfine Interact. 215, 69 (2013)

[14] B.V. Batyunya et. al, JINR Preprint P1-87-849 (1987) 